\author{
Justus G. Garweg \\ Matthias Boehnke
}

\section{The antibody response in experimental ocular toxoplasmosis}

Received: 23 April 2005

Revised: 24 November 2005

Accepted: 10 January 2006

Published online: 6 April 2006

C) Springer-Verlag 2006
J. G. Garweg $(\bowtie)$

Swiss Eye Institutes, Gossetstrasse 43,

3084 Wabern, Switzerland

e-mail: justus.garweg@hotmail.com

Tel.: +41-31-632-9565

Fax: +41-31-632-8539

M. Boehnke

Hamburg Institute of Ophthalmology,

Rothenbaumchaussee 123 ,

20149 Hamburg, Germany

\begin{abstract}
Background: The dynamics of the humoral immune response in ocular toxoplasmosis (OT) are poorly understood. We therefore investigated this process in a rabbit model of the disease. Materials and methods: Of 24 infection-naïve adult rabbits, 12 were left untreated and 12 were systematically infected with 5,000 tachyzoites of the non-cystforming BK strain of Toxoplasma gondii. Three months later, all rabbits were inoculated transvitreally with 5,000 tachyzoites of Toxoplasma gondii. Paired samples of aqueous humor and serum were analyzed temporally for their total and specific IgG contents. Results: In infectionnaïve rabbits with primary OT, specific IgG reached detectable levels in the inoculated eyes between 5 and 15 days after inoculation. In infectionimmunized rabbits with secondary OT, a significant increase in specific $\operatorname{IgG}$ was regularly detected after 5 days. The antibody ratio $\mathrm{C}$ was
\end{abstract}

diagnostic ( $\geq 3$ ) from day 15 onward in primary OT and from day 21 onward in secondary OT. In the uninfected partner eyes, the antibody ratio $\mathrm{C}$ was found sporadically diagnostic from day 15 onward in primary OT, but at no time in secondary OT. Specific IgG persisted both locally and in the serum until the end of the monitoring period (100 days).

Conclusion: Our findings relating to the rabbit model of OT reveal three features of clinical relevance: a diagnostic window precedes the establishment of a humoral immune response; specific antibodies persist long after the cessation of disease activity; and in primary OT, the antibody ratio $\mathrm{C}$ may also increase in the uninfected partner eye.

Keywords Ocular toxoplasmosis . Toxoplasma gondii - Aqueous humor . Antibodies - Rabbit - BK strain · Animal study - ELISA · Falselynegative results $\cdot$ Laboratory testing

\section{Introduction}

Ocular toxoplasmosis is the most frequent cause of posterior uveitis $[26,36]$ and of the associated visual impairment in immunocompetent individuals [37]. The diagnosis of this etiology is based upon characteristic clinical manifestations [5, 37, 48]. However, atypical clinical pictures also exist, and these are not so readily identified, even by experienced practitioners [8, 11, 27]. This circumstance raises questions as to the sensitivity and specificity of the clinical diagnosis, which, in the absence of a sufficiently sensitive laboratory test for the disease, is still regarded as the gold standard [45].

Various serological tests have been approved to confirm a past systemic infection. These are based on the detection of specific IgG in the serum in the absence or, more rarely, in the presence of low levels of specific $\operatorname{IgM}[25,33]$. Consequently, although the absence of specific $\mathrm{IgG}$ permits an exclusion of ocular toxoplasmosis, its presence does not confirm the existence of the disease. 
In atypical cases, a speedy laboratory confirmation of the suspected clinical diagnosis is necessary [38], and this may be achieved in $60-80 \%$ of cases by a combined analysis of the aqueous humor and serum. This is based on the detection of local production or at least a locally increased concentration of specific IgG and, more rarely of IgA or $\operatorname{IgM}[3,11,20,32,33]$. The sensitivity and the specificity of this combined aqueous humor and serum analysis have been established only recently [52]. From a practical point of view, the crux of the matter in human studies is a correct clinical diagnosis, which is still deemed to be the gold standard for clinical laboratory testing. The predictive value of the clinical diagnosis depends greatly on the clinician's experience. But since the sensitivity of the laboratory tests is still unsatisfactory, the clinical picture and the course of the disease under therapy may be the only available pointers for a diagnosis. The strength or weakness of the clinical diagnosis may contribute to the variable sensitivity (i.e., confirmation frequency) of the results gleaned from combined aqueous humor and serum analyses, which ranges from 50 to $85 \%[7,20,29,35$, 42]. Moreover, the proportion of false-negatives and, worse still in terms of the treatment consequences, of falsepositives, is as yet unknown.

The difficulty in establishing the predictive power of a laboratory test in the clinical setting may be exemplified with the Goldmann-Witmer index, which is the oldest and probably still the most meaningful laboratory indicator of ocular toxoplasmosis. Even now, 50 years after the introduction of this test for uveitis by Goldmann and Witmer [23], the time dependence of the findings, although recognized [20], has not been systematically investigated. A cut-off value for the interpretation of data was set in 1966 on the basis of clinical evidence [10] and has since been corrected according to experience [28, 29]. But a high proportion of clinically typical cases still fail to manifest a sufficiently large local IgG response to permit laboratory confirmation of the disease. Hence, the inclusion of other tests, such as the determination of antibody avidity $[18,50$, 53], the detection of specific $\operatorname{IgA}$ [20], and immunoblotting $[21,52]$, has been advocated to improve the diagnostic yield of the antibody analysis. Finally, qualitative and quantitative measurements of local antibody production are frequently inconsistent [21]. Although the synthesis of oligoclonal $\mathrm{IgG}$ is detected in up to $87 \%$ of the aqueous humor samples of ocular toxoplasmosis patients, there is also evidence of a polyspecific immune response in unrelated chronic inflammatory diseases, such as multiple sclerosis [43]. These discrepancies, and our inability to rationalize them, reflect our poor understanding of the pathophysiological mechanisms underlying local antibody production.

The present experimental study was undertaken with a view to shedding further light on several principal aspects of local $\mathrm{IgG}$ production in ocular toxoplasmosis using a rabbit model of the disease. We wished to follow the time course of local specific antibody production, bilaterally, in naïve and systematically infection-immunized rabbits, which were subsequently inoculated with tachyzoites of Toxoplasma gondii in only one eye.

\section{Materials and methods}

\section{Animal experiments}

All animal experiments were approved by the local and institutional Animal Ethics Committee, and were performed according to the Principles of laboratory animal care (NIH publication No. 85-23, revised 1985) and to the ARVO guidelines for animal research under the surveillance of the local Public Veterinary Health Authorities. Experimental ocular toxoplasmosis was induced in infection-naïve rabbits or secondarily induced by intraocular reinfection in systemically infection-immunized animals as previously reported [19], using the same strain of Toxoplasma gondii tachyzoites.

Twenty-four seronegative rabbits obtained from a local breeder were used in this study. They were of both sexes, were at least 4 months old, and had a body weight ranging from 3.5 to $5 \mathrm{~kg}$. Twelve of the rabbits were left untreated until the time of ocular infection (group 1). The other 12 rabbits (group 2) were each infected subcutaneously with 5,000 tachyzoites of the virulent, non-cyst-forming BK strain of Toxoplasma gondii. Between days 8 and 28, they received intramuscular injections of clindamycin $(20 \mathrm{mg} /$ $\mathrm{kg}$ of body weight/day) to prevent a lethal course of events. A 3-month period was then allowed for the infection to settle down. At the end of this period, both eyes of all rabbits (groups 1 and 2) underwent dilated pupil fundoscopy in order to exclude any ocular pathology. The left eye was infected transvitreally with tachyzoites of the BK strain of Toxoplasma gondii under indirect ophthalmoscopic control. The right eye of each rabbit served as an uninfected control. Between days 8 and 28, all animals received an intramuscular injection of clindamycin, again to prevent a lethal course of events. The rabbits were subjected to regular clinical examination. Blood and aqueous humor samples from both eyes were collected under conditions of general anesthesia, prior to infection, and on days $5,10,15,21,28,35,42,60,90$, and 100 . All samples were processed immediately as described below, and then stored at $-20^{\circ} \mathrm{C}$ until required for the antibody analyses, which were run simultaneously.

\section{Methods}

\section{Sample collection and work-up}

Aliquots of $150-250 \mu \mathrm{l}$ of aqueous humor were collected by anterior chamber puncture using a 30-gauge needle and 
a tuberculin syringe. Each sample was centrifuged immediately after its collection. The sediment was used for the amplification of DNA (data not presented here) and the supernatant for the antibody analysis. Serum was obtained from a blood sample after clotting and centrifugation. This was likewise used for the antibody analysis.

\section{Total rabbit $\operatorname{Ig} G$}

Total rabbit IgG was quantified using a customized rabbit IgG ELISA test system under standard conditions. A triplicate set of controls with a given antibody concentration was run in parallel with the test samples on each test plate to internally equilibrate the system. The test samples were run in duplicate and the average measurement was used for subsequent calculations.

\section{Specific rabbit anti-toxoplasma $\operatorname{Ig} G$}

Specific rabbit anti-toxoplasma IgG was quantified using a special customized ELISA system, which was developed for the analysis of a larger number of animal samples than is possible using commercial test systems. Prior to a definitive establishment of this test system, we compared the results yielded with those obtained using a commercial Toxoplasma IgG ELISA test system (Platelia Toxo; SanofiDiagnostics Pasteur, Marnes la Coquette, France), which we employed for routine testing. Freshly frozen tachyzoites from the culture supernatants of infected HEP-2 cell lines (passages 7-15) were added to the coating buffer $(0.1 \mathrm{M}$ $\mathrm{Na}_{2} \mathrm{CO}_{3}$ and $0.1 \mathrm{M} \mathrm{NaHCO}_{3}[\mathrm{pH} 9.6]$ ) at a concentration of $2.5 \times 10^{4} / \mathrm{ml}$ after thorough washing and disruption by freezing and thawing. All test plates were coated in parallel and stored in a vacuum at $-20^{\circ} \mathrm{C}$ for less than 6 weeks prior to the analyses. They were then washed with blocking reagent (phosphate-buffered saline $[\mathrm{pH}$ 7.4; Hospital Pharmacy, Inselspital Bern, Switzerland] containing 0.1\% Tween and 2\% fetal calf serum). Duplicate test samples were diluted 1:100 in phosphate-buffered saline ( $\mathrm{pH} 7.4)$ and then incubated with the test plates for $2 \mathrm{~h}$ at $37^{\circ} \mathrm{C}$. The test plates were thoroughly rinsed and then incubated with alkaline-phosphatase-conjugated goat anti-rabbit $\operatorname{IgG}$ and naphthol AS as a substrate. The plates were scanned with an ELISA reader (SpectraMax 250; Molecular Devices, Sunnyvale, CA, USA) to quantify the information. For calibration purposes, standard rabbit serum containing specific IgG at concentrations of approximately 6, 60, 240, and 1,000 WHO unit equivalents (IU) was run in triplicate on each test plate. The reproducibility of the results was confirmed by running a randomly selected portion of the test samples (approximately 10\%) on a separate occasion. The lower and upper cut-off levels for this assay were set at 4 and 4,000 IU respectively.
Whilst setting up this study, we attempted to quantify not only IgG but also IgM and IgA. Numerous commercially available products of specific rabbit $\operatorname{IgM}$ and $\operatorname{IgA}$ were tested, but none of these were sufficiently specific to permit their unique determination without binding to rabbit IgG.

The Goldmann-Witmer coefficient (antibody ratio C) was calculated using Desmonts' formula ([10]): antibody ratio $\mathrm{C}=$ [specific $\mathrm{IgG}$ in the aqueous humor/total $\mathrm{IgG}$ in the aqueous humour]/[specific IgG in the serum/total IgG in the serum]. An antibody ratio $\mathrm{C}$ of more than or equal to 3 was assumed to be indicative of local antibody production.

In the present study, data are described using descriptive measures such as the median, minimal and maximal values, and the standard deviation. These are displayed graphically when appropriate. The levels of total IgG in the aqueous humor and serum are presented in a single figure. To avoid cluttering, the minimum and maximum values and the standard deviations have been omitted.

The main object of this non-comparative study was to describe temporal changes in antibody concentration. Hence, for a given set of data, the results obtained at each time point were statistically compared only with the base-line value (day 0). For this comparison (Student's $t$ test), the data were assumed to be normally distributed. The level of statistical significance was set at $p<0.05$.

\section{Results}

Although prophylactic measures were taken to prevent a lethal course of events, 3 of the 12 infection-immunized rabbits (secondary ocular toxoplasmosis [group 2]) and 2 of the 12 infection-naïve rabbits (primary ocular toxoplasmosis [group 1]) died within 14 days of inoculation with tachyzoites of Toxoplasma gondii.

In all of the remaining animals, retinochoroiditis with marked vitreal infiltration became apparent 3-5 days after transvitreal inoculation. Systemic signs of infection, such as an increased body temperature and inappetence were manifested from days 5 to 7 onward.

In both the primary ocular toxoplasmosis group (group $1 ; n=10)$ and the secondary ocular toxoplasmosis group (group $2 ; n=9$ ), a diagnostic window describing the time elapsing between the onset of clinical symptoms and the induction of local antibody production was apparent before the aqueous humor levels of Toxoplasma-specific IgG began to increase significantly in either the inoculated or the partner eye (Fig. 1a-d). In group 1 (primary ocular toxoplasmosis), a statistically significant increase in specific IgG above the detection threshold began on day 15 in the infected eye and on day 49 in the uninfected partner eye $(p<0.05)$, but in single instances, it was detectable from day 5 onwards. In group 2 (secondary ocular toxoplasmosis), the increase in specific $\operatorname{IgG}$ began on day 5 in the infected eye $(p<0.05)$. In the uninfected partner eye, the local level of specific IgG did not increase significantly at 
A

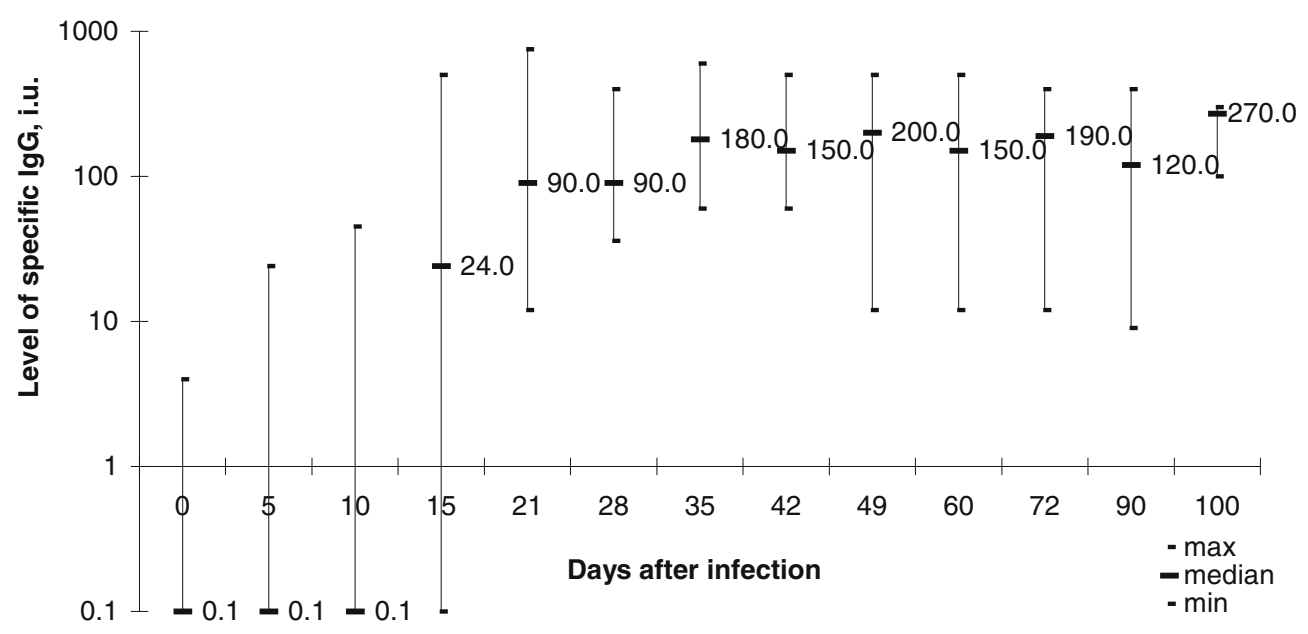

B

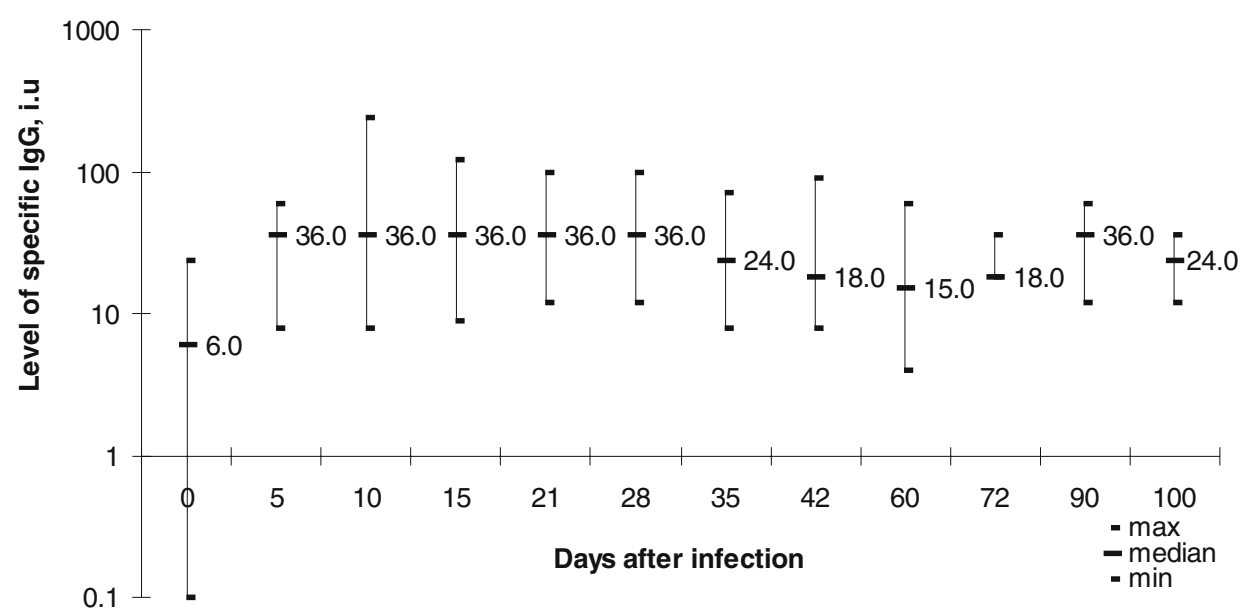

C

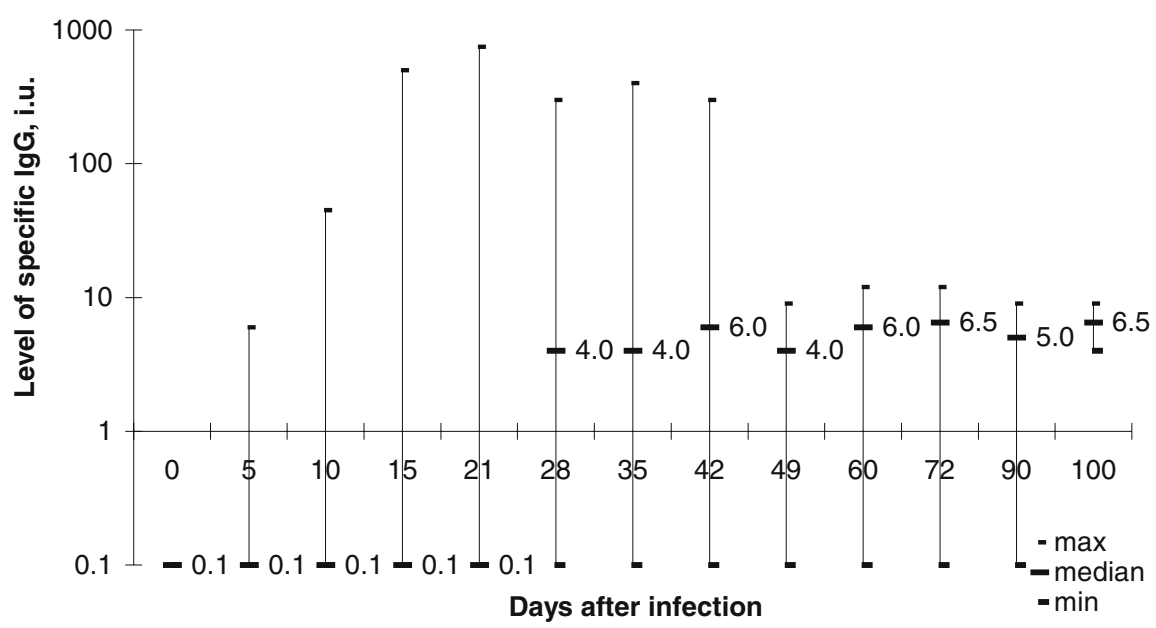


Fig. 1 (continued)
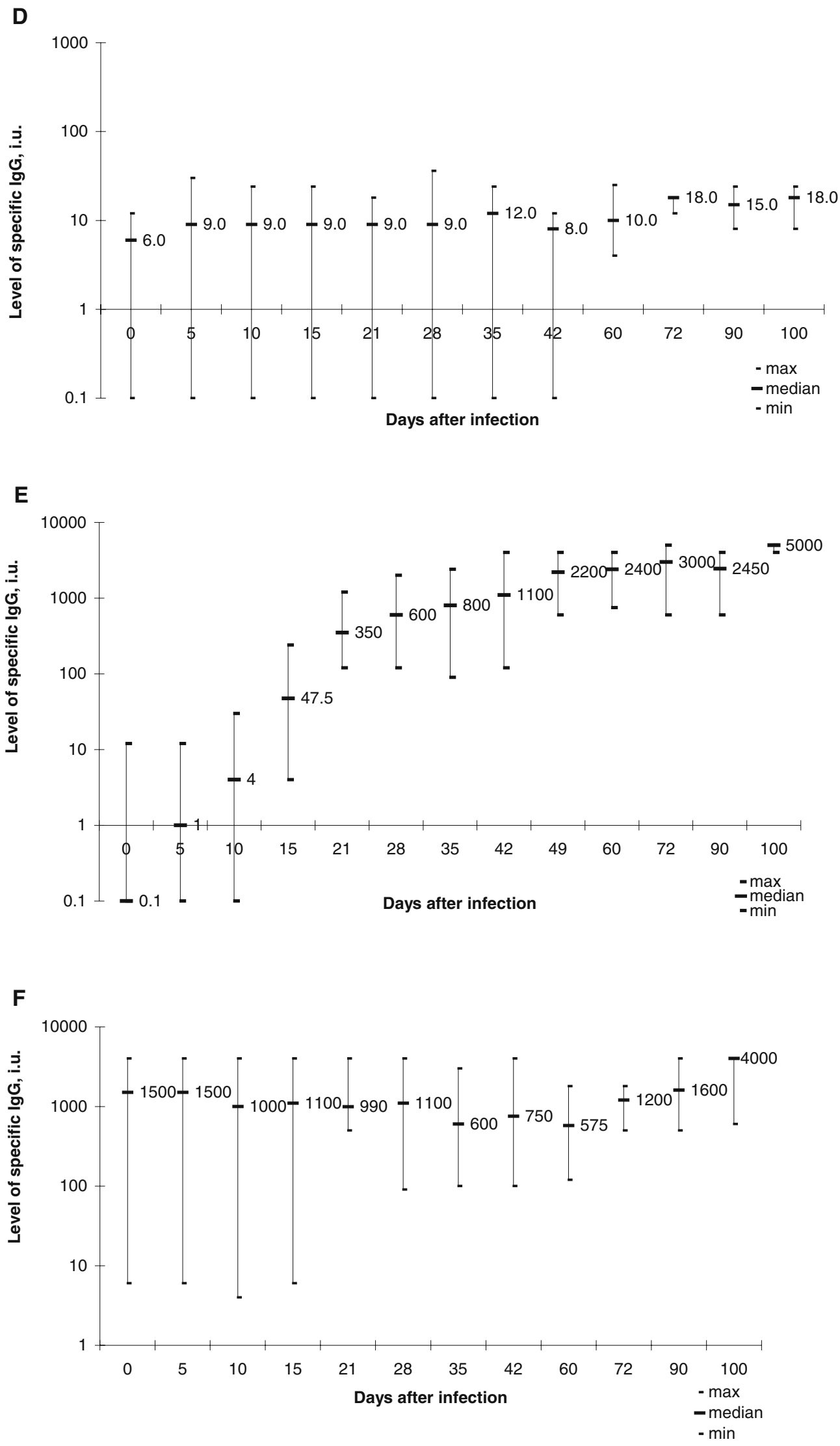
4Fig. 1 Median, minimal, and maximal values (expressed in International Unit equivalents [IU] of Toxoplasma-specific IgG) are presented. Values near the detection limit of the anti-Toxoplasma IgG ELISA test system were arbitrarily set at $0.1 \mathrm{IU}$ to permit their graphical representation. a Activation of Toxoplasma-specific IgG production in the aqueous humor of the inoculated eye of infectionnaïve rabbits with primary ocular toxoplasmosis (group $1 ; n=10$ ). Specific IgG reached detectable levels in the inoculated eyes between 5 and 15 days after inoculation, attaining statistical significance from day 15 onwards. b Activation of Toxoplasmaspecific IgG production in the aqueous humor of the inoculated eye of infection-immunized rabbits with secondary ocular toxoplasmosis (group 2;n=9). Compared with the base-line level, the increase in specific IgG attained statistical significance from day 5 onwards. c Activation of Toxoplasma-specific IgG production in the aqueous humor of the uninfected partner eye of infection-naïve rabbits with primary ocular toxoplasmosis (group $1 ; n=10$ ). Specific IgG levels in the uninoculated eyes lay regularly below those of inoculated eyes, sporadically reaching detectable levels between 5 and 21 days after inoculation and attaining statistical significance from day 49 onward. d Activation of Toxoplasma-specific IgG production in the aqueous humor of the uninfected partner eye of infectionimmunized rabbits with secondary ocular toxoplasmosis (group 2; $n=9)$. Compared with the base-line level, the increase in specific IgG never attained statistical significance. e Activation of Toxoplasmaspecific IgG production in the serum of infection-naïve rabbits with primary ocular toxoplasmosis (group $1 ; n=10$ ). Compared with the base-line level, the increase in specific IgG attained statistical significance from day 10 onwards. Typically for naïve rabbits, low levels of naturally occurring unspecific serum antibodies against Toxoplasma gondii are detectable. f Activation of Toxoplasmaspecific IgG production in the serum of infection-immunized rabbits with secondary ocular toxoplasmosis (group $2 ; n=9$ ). Compared with the base-line level, the level of specific IgG did not change significantly during the monitoring period

any point in time $(p>0.05)$. In group 1 (primary ocular toxoplasmosis), the evolution of this rise in the serum level of specific IgG paralleled the local course (Fig. 1e). In group 2 (secondary ocular toxoplasmosis), the serum levels of specific IgG did not change during the entire monitoring period (100 days; Fig. 1f).

The activation of a humoral immune response was also reflected in the levels of total IgG. In group 1 (primary ocular toxoplasmosis), the level of total IgG had risen after 3 weeks by less than 2 -fold in the serum, by 3 -fold in the aqueous humor of the uninfected partner eye, and by more than 20 -fold in the aqueous humor of the inoculated eye (Fig. 2a). In group 2 (secondary ocular toxoplasmosis), the level of total IgG did not change either in the serum or in the aqueous humor of the uninfected partner eye. But in the inoculated eye, the local level rose 8- to 10-fold after 10 days (Fig. 2b). This increase may be accounted for by local specific IgG production and by an uveovascular barrier breakdown.

In group 1 (primary ocular toxoplasmosis), the antibody ratio $\mathrm{C}$ was indicative of local $\operatorname{IgG}$ production $(\geq 3)$ in single instances from day 5 to day 42 in the inoculated eye, and from days 15 to 42 in the uninfected partner eye (Fig. 3a). In group 2 (secondary ocular toxoplasmosis), the antibody ratio $\mathrm{C}$ values were significantly lower. For the inoculated eye, they were indicative of local $\mathrm{IgG}$ produc- tion ( $\geq 3$ ) from day 21 to day 72; whereas for the uninfected partner eye, they were at no time indicative (Fig. 3b).

After systemic (subcutaneous) infection of infectionnaïve rabbits ( $n=12$; group 2 prior to intraocular infection), the levels of both specific and total $\mathrm{IgG}$ rose significantly $(p<0.05)$ and in parallel from day 15 in the serum and from day 21 in the aqueous humour (Fig. 4a,b), which is 5 or more days later than after ocular infection. The level of total $\mathrm{IgG}$ in the serum rose 2-fold, which corresponds to the rise observed after the ocular infection of naïve animals (primary ocular toxoplasmosis (group 1)) at a later stage. The level of total IgG in the aqueous humor of these clinically uninfected eyes (right and left) rose 3-fold (Fig. 4b), which indicates a moderate compromise of the uveovascular barrier. By 2 months, the situation had normalized (Fig. 4b). In the absence of clinically detectable lesions, the antibody ratio $\mathrm{C}$ became indicative $(\geq 3)$ at least once between days 21 and 60 in 9 eyes of the 6 surviving rabbits and remained negative throughout in the 6 eyes of the 3 remaining rabbits (Fig. 4c). Correction for breakdown of the uveovascular barrier would have increased the number of indicative antibody ratios.

\section{Discussion}

The present study was undertaken with a view to improving our understanding of the dynamics of the humoral immune response in ocular toxoplasmosis, and to shedding further light on the factors contributing to its sensitivity and specificity. Regarding the underlying principles of the humoral immune response, the clinically most interesting findings of our study are the following:

- The existence of a diagnostic window embracing infection, clinical symptoms, and the local immune response, which is more pronounced in secondary (recurrent) than in primary ocular toxoplasmosis (Fig. 1a,b)

- The antibody ratio $C$ values suggest the existence of plasma-cell homing after 15 days in the uninfected partner eye of rabbits with primary ocular toxoplasmosis (Fig. 3a), but not in those with secondary ocular toxoplasmosis (Fig. 3b). It should be borne in mind, however, that although a measurable rise in specific IgG production was discernible, the increase was not significant

- An increase in the serum level of specific IgG was detected in infection-naïve rabbits (primary ocular toxoplasmosis; Fig. 1e), but not in infection-immunized animals (secondary ocular toxoplasmosis; Fig. 1f), which is in accordance with clinical experience

- A breakdown of the uveovascular barrier, with a relative increase in the level of total $\mathrm{IgG}$, was observed 
Fig. 2 a Median total $\mathrm{IgG}$ levels in infection-naïve rabbits with primary ocular toxoplasmosis (group $1 ; n=10$ ). Breakdown of the uveovascular barrier is indicated by a more than 20 -fold increase in the level of total $\mathrm{IgG}$ in the aqueous humor of the infected (left) eye and by up to a 3-fold one in the uninfected (right) eye. b Median total IgG levels in infectionimmunized rabbits with secondary ocular toxoplasmosis (group $2 ; n=9)$. Breakdown of the uveovascular barrier is indicated by up to a 10 -fold increase in the level of total $\mathrm{IgG}$ in the aqueous humor of the infected (left) eye and by a 2-fold one in the uninvolved (right) eye
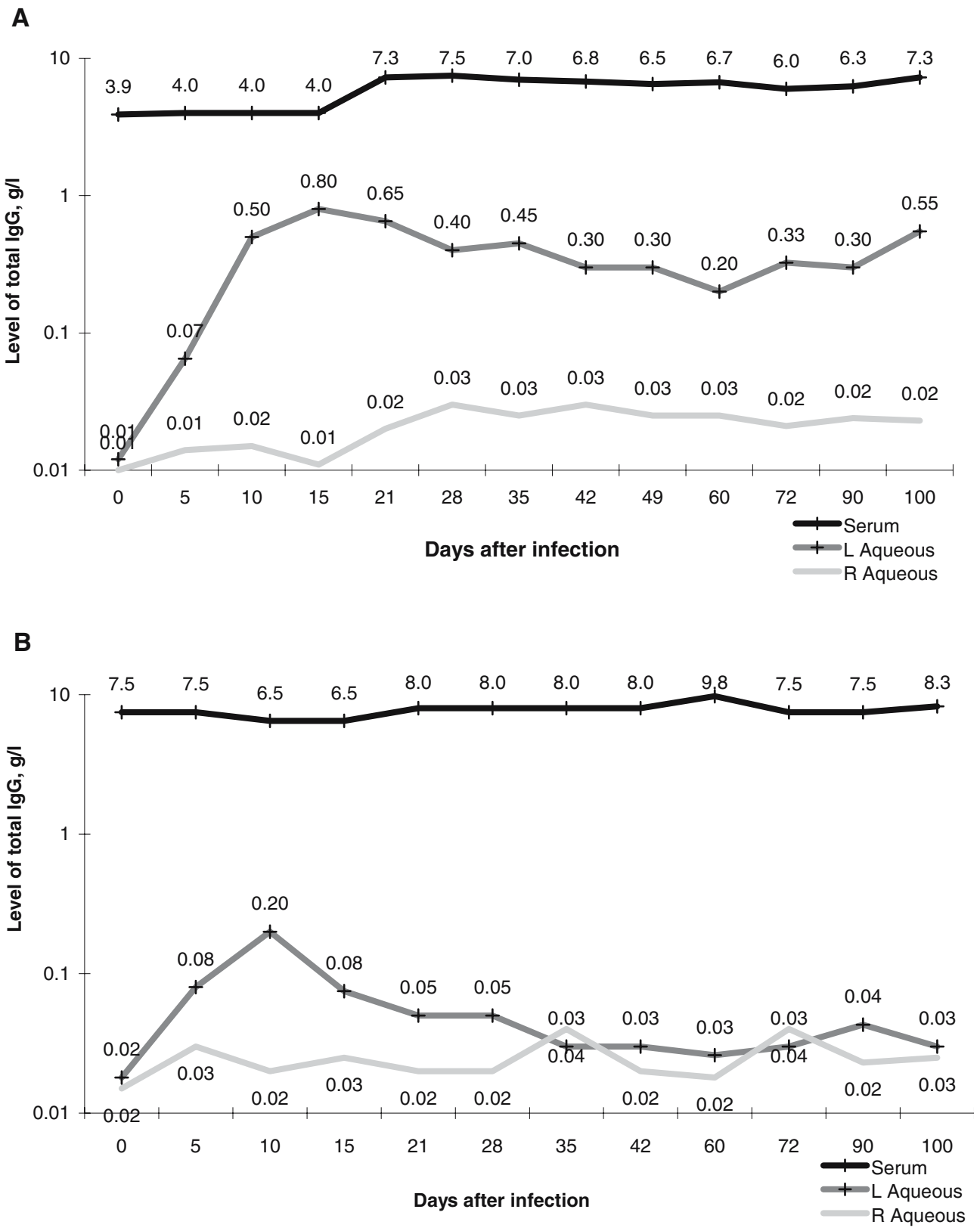

in primary, but not in secondary ocular toxoplasmosis (Fig. 2a,b)

- In systemic toxoplasmosis, there was evidence of an unremarkable, site-specific increase in specific and total IgG (Fig. 4a,b), which resulted in the sporadic attainment of indicative antibody ratios (Fig. 4c)

In atypical cases of ocular toxoplasmosis, a laboratory confirmation of the suspected clinical diagnosis is recommended $[3,4,10,20,22,28,29,40-42,44]$. This is based on the detection of Toxoplasma gondii DNA within either the aqueous humor or, preferably, the vitreous $[1,2$, $7,16,52]$, and of local specific antibody production [20]. However, our poor understanding of the dynamics of local antibody production and the absence of a single, sufficiently sensitive and specific laboratory test [52] have not encouraged the broad clinical acceptance of a combined serum and aqueous humor antibody analysis in suspected cases of ocular toxoplasmosis. On the other hand, the usefulness of performing serological tests in pregnant women and in newborn infants to establish the existence of a congenitally transferred infection [6], and the recognition of a compartmentalized humoral immune response in ocular disease [32], are generally accepted.

Although it could be argued that our experimental rabbit model does not closely resemble the human situation quantitatively, it is nevertheless useful in testing some of 
Fig. 3 a Goldmann-Witmer antibody coefficients (antibody ratio $\mathrm{C}$ ) for the infected (left) and the uninvolved partner (right) eyes of infection-naïve rabbits with primary ocular toxoplasmosis (group $1 ; n=10$ ). Median values are represented together with the standard errors (bars). Note that diagnostically indicative values $(\geq 3)$ are achieved between days 5 and 42 for the infected eye and sporadically between days 15 and 42 for the uninfected partner eye. b Goldmann-Witmer antibody coefficients (antibody ratio C) for the infected (left) and the uninvolved partner (right) eyes of infection-immunized rabbits with secondary ocular toxoplasmosis (group 2;n=9). Median values are represented together with the standard errors (bars). Note that diagnostically indicative values $(\geq 3)$ are achieved between days 21 and 72 for the infected eye, but at no time for the uninfected partner eye
A
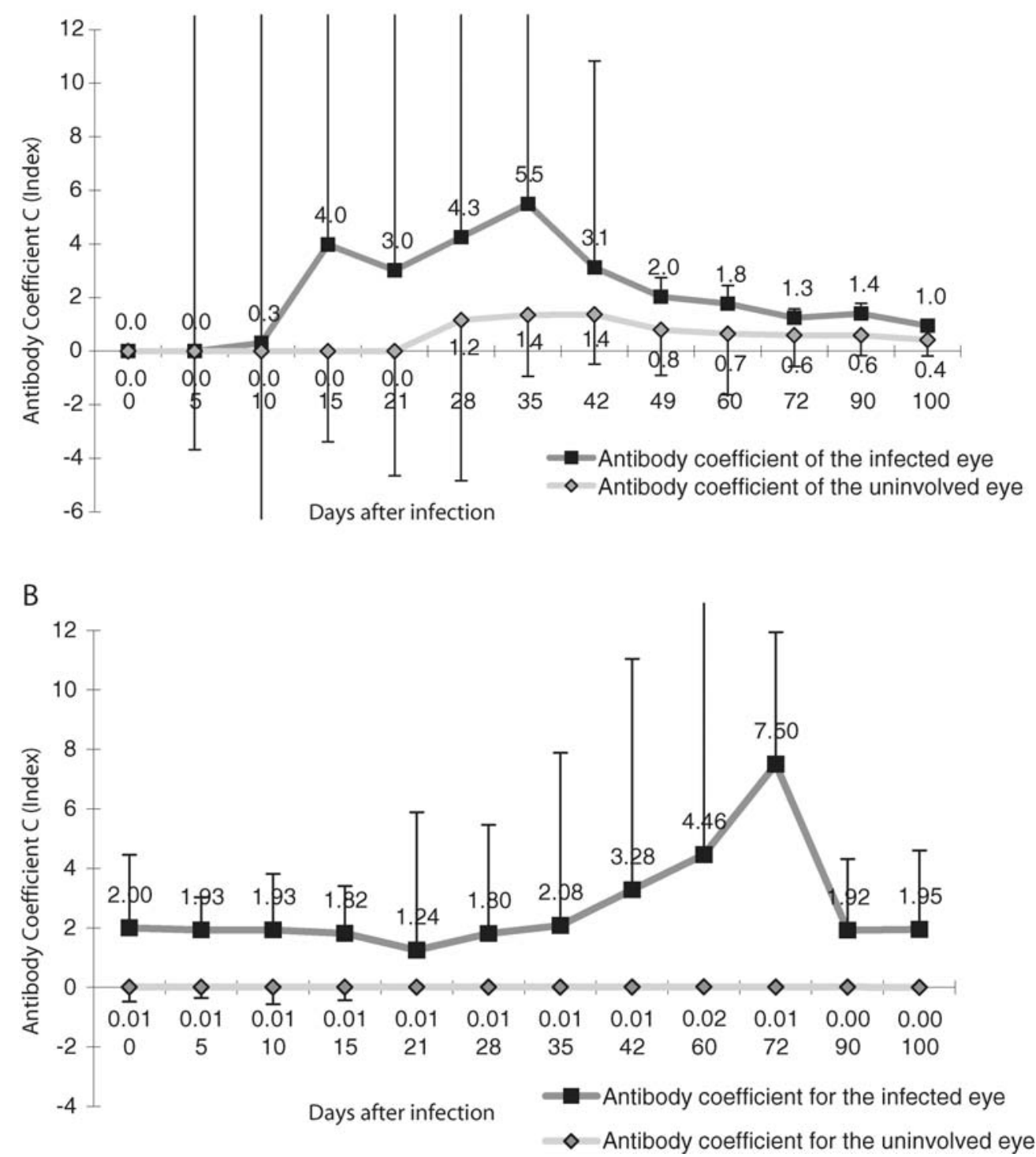

the principles governing the humoral immune response. In a previous study comparing outbred and inbred rabbits, antigen stimulation was reported to increase oxidative stress, which provoked a compensatory and adaptive upregulation of superoxide dismutase for up to 30 days. Both responses were more pronounced in the inbred than in the outbred rabbits [46]. The 30-day time course corresponds with the establishment of a systemic humoral immune response, as shown in our model and by Langford et al. [34]. However, great caution must be exercised in drawing quantitative conclusions, since many unconsidered factors, such as homozygosity, may interfere with the quantitative immune response. Tanchev et al. have demonstrated that a simultaneous immunomodulation may elicit different antibody responses [49]. Such a situation could arise from a clinically non-evident bacterial or coccidial gastrointestinal superinfection of the systemically seriously impaired rabbits after primary Toxoplasma infection and parenteral antibiotic therapy. The humoral immune response is obviously paralleled by the cellular one, which is manifested 14 days after subretinal xenogenic cell transplantation [15].

Obviously, the intraocular transvitreal route of infection does not correspond to the natural one. This may be the weakest aspect of our model, since the route of infection has a substantial impact not only on the establishment of the cellular immune response, but also on the quantitative humoral one [24]. Nevertheless, there is no evidence that the route of infection interferes with the principal observations of our study, such as the time course of the immune response or the response of the partner eye. Hence, 
Fig. 4 a Activation of Toxoplasma-specific IgG production in the aqueous humor and serum of naïve rabbits after their systemic infection with Toxoplasma gondii $(n=12)$. Compared with the base-line level, the increase in specific IgG attained statistical significance from day 15 onward in the serum and from day 21 onward in the aqueous humor. Median values (expressed in IU of Toxoplasmaspecific IgG) are presented. Values near the detection limit of the anti-Toxoplasma IgG ELISA test system were arbitrarily set at 0.1 to permit their graphical representation. b Median total IgG levels in naïve rabbits after their systemic infection with Toxoplasma gondii $(n=12)$. Moderate impairment of the uveovascular barrier is indicated by up to a 3 -fold increase in the level of total $\mathrm{IgG}$ in the aqueous humor of each eye, compared with only a 2fold one in the serum, which attained statistical significance (relative to the base-line level) on day 21 . This finding reflects not only the activation of plasma cells, but probably also the impact of frequent anterior-chamber punctures. The situation normalized after 2 months. c Goldmann-Witmer antibody coefficients (antibody ratio $\mathrm{C}$ ) for both eyes in naïve rabbits after their systemic infection with Toxoplasmosis gondii $(n=12)$. Median values are represented together with the standard errors (bars). Significant increases $(p<0.05)$ and diagnostically indicative values $(\geq 3)$ are achieved between days 21 and 60 for single eyes in the absence of clinically detectable lesions. Correction for uveovascular barrier damage would have yielded even higher values
A

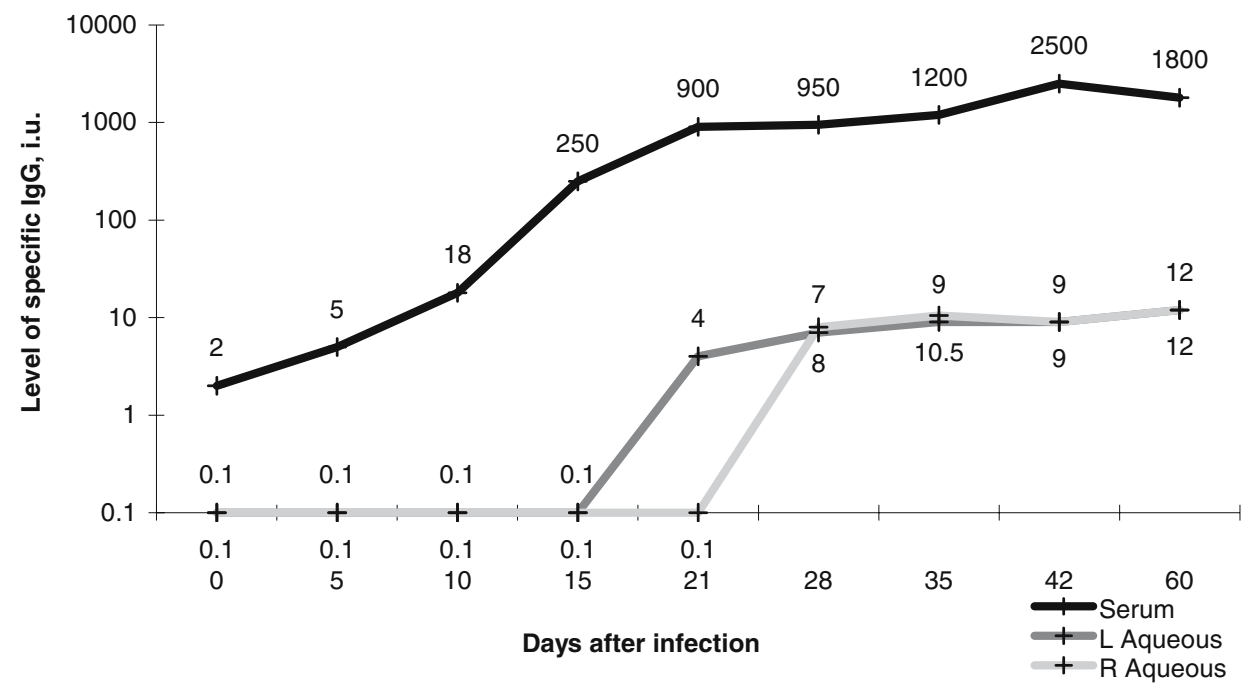

B

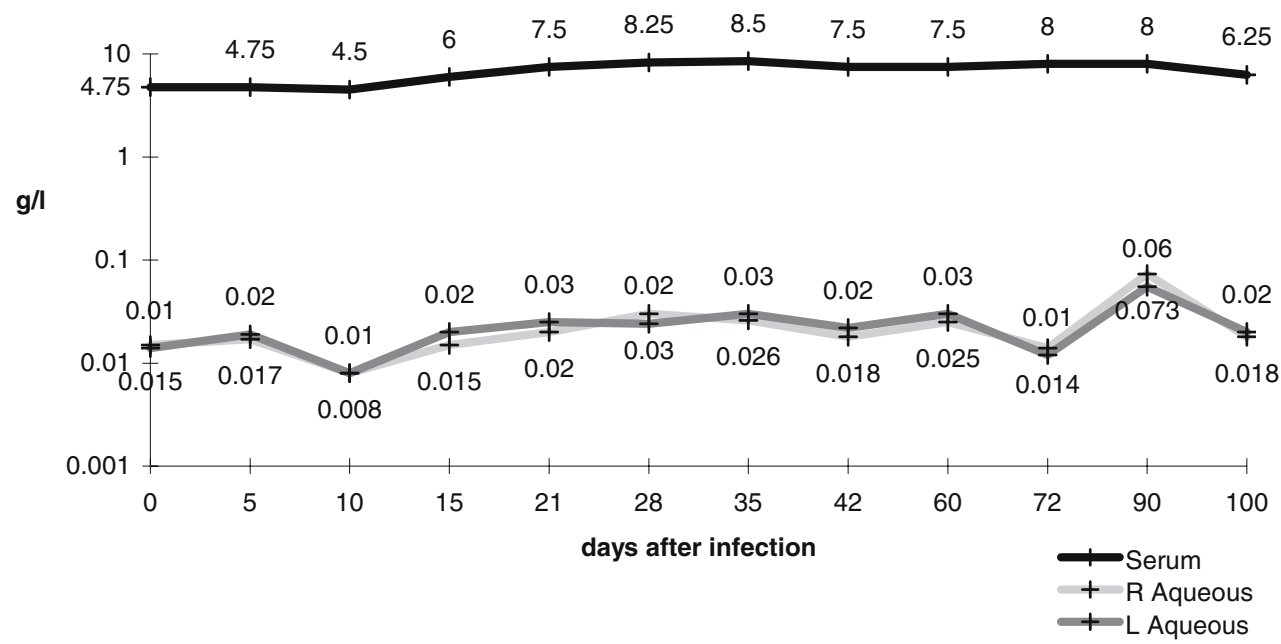

C

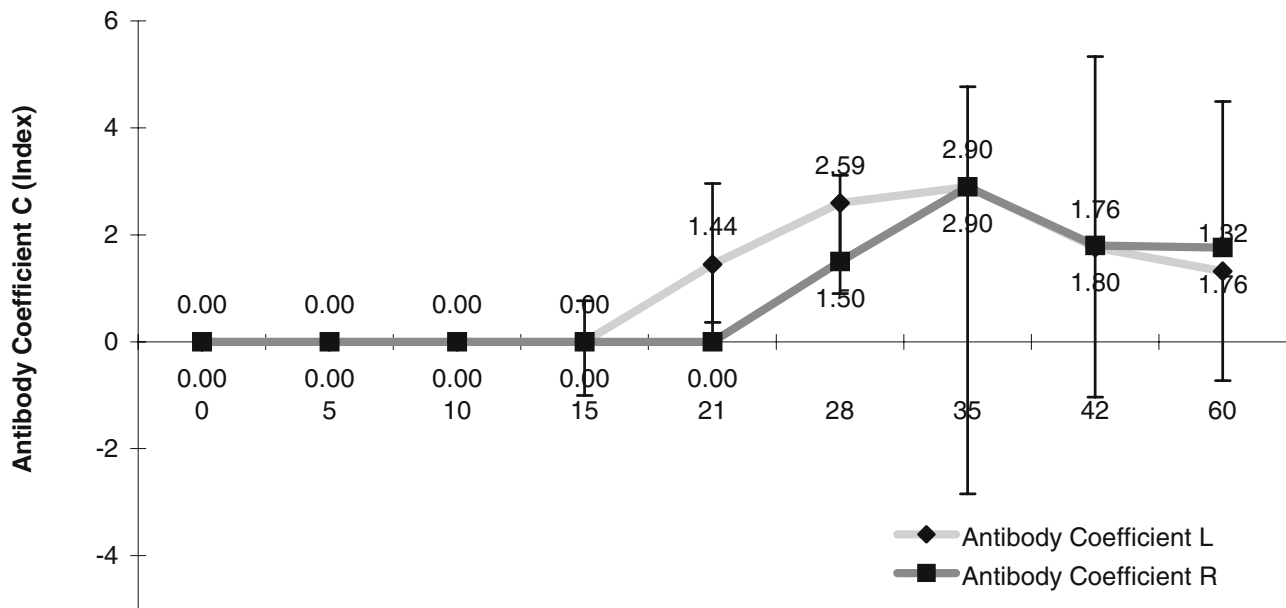

Days after infection 
we are confident that our results will be helpful in understanding and interpreting clinical laboratory data.

One other critical issue of our study relates to the frequency of anterior-chamber puncturing. Whether these interventions underlie the site-specific relative increase in total aqueous humor IgG above the serum level is open to discussion (Fig. 4b), since they do not account for the indicative antibody ratio values observed in more than onethird of the eyes in the absence of clinically manifest chorioretinal lesions (Fig. 4c). Also, the cause of the high inter-individual range of the results cannot be obviously explained. But they could reflect the relatively low concentrations of total and specific IgG within the aqueous humor (compared with those in the serum). These hover around the lower cut-off detection level for the ELISA test system. Serum samples, on the other hand, must be diluted. Despite the limitations of our rabbit model, a more appropriate alternative has not been described to date [9].

A comparison of our results with existing clinical laboratory findings reveals several common features: apart from confirming a humoral infection-associated immunity to Toxoplasma gondii, a serum antibody analysis does not otherwise contribute to the diagnosis $[20,25]$. If clinically indicated, laboratory testing is useful only if it involves a parallel analysis of the humoral immune response in both the aqueous humor and the serum [10, 29]. But even a combined analysis will not reveal the expected information if the samples are withdrawn too early [20], a clinical experience that is also supported by the present findings.

Local antibody production is deemed to be underway if the antibody ratio $C$ lies above $3[10,23,29,30]$, and it still represents the hallmark for a laboratory confirmation of ocular toxoplasmosis. Depending on the case selection criteria, the sensitivity of this index as a diagnostic tool lies between 60 and $85 \%$ [3, 4, 7, 10, 13, 20, 22, 29, 42, 52], with a specificity approaching $90 \%$ in persons of European or North American origin if combined with other serological analyses, as reported by Villard et al. [52]. Referring to the sensitivity of antibody ratio $\mathrm{C}$, it has to be kept in mind that cut-off values may differ between single studies. And, surprisingly, a generally accepted formulation for determination of antibody ratio $\mathrm{C}$ values has also not been agreed on. In the above-mentioned study by Villard, for example, the integrity of the blood-aqueous barrier was assessed not by comparing anti-Toxoplasma-specific antibodies with the total IgG fraction, as is usual, but with antibodies against the Mumps virus. These antibodies may attain a serum prevalence of $100 \%$ in a healthy population without inducing chorioretinitis. Hence, their presence in the aqueous humor reflects a passive transudation from the serum through an impaired blood-retina barrier. This modification was first suggested by Turunen et al. [50], and the sensitivity and specificity thereby achieved are possibly better than when the comparison is based on the total IgG level. However, we have been unable to identify a rabbit
ELISA kit of corresponding sensitivity and specificity. Nevertheless, a confirmation of antibody production within the eye indicates that the site of inflammatory activity may be localized to this organ, which would be expected for the congenitally-contracted disease. In patients with an acquired form of the disease, clinical manifestations may be restricted to the eye even if the infection is systemic [31, $32,39]$. This aspect has not been addressed with this model, although the positive local antibody indices found even in systemic disease without evident ocular involvement, together with clinical experience, argue against the assumption of an absolute compartment-specific humoral immune response [32].

Several aspects relating to the diagnosis of ocular toxoplasmosis are still under debate. One of these concerns the time elapsing between the onset of clinical symptoms and the induction of local antibody production. In twothirds of patients who register negative for specific antibody production at the first clinical presentation, evidence of induction is revealed at a later stage [20]. This finding is consistent with earlier results of our own pertaining to the experimental rabbit model of ocular toxoplasmosis [19]. These revealed that local antibody production did not begin until 2 weeks after inoculation and 10 days after a marked infiltration of the vitreous. Humoral and/or cellular immunotolerance phenomena could conceivably account for the failure to detect specific antibodies within the aqueous humor [54]. Numerous other and as yet unidentified factors may be relevant, including the virulence of the parasite, its route of acquisition and immunogenetic influences [17]. These might contribute to intraocular infection-associated inflammatory processes in general $[14,47]$ and to ocular toxoplasmosis in particular $[12,51]$. However, these possibilities have not been systematically investigated. Another debatable issue is the persistence of local antibody production after the cessation of disease activity, and the possible presence of specific antibodies in uninvolved eyes. Our own data (Fig. 1a-f) as well as clinical observations by other investigators [13] support these contentions.

Although we are well aware that our experimental rabbit model of ocular toxoplasmosis does not closely resemble the human ocular disease, and that the dynamics of the specific humoral immune response may differ from the human situation, our data nevertheless afford an insight into some of the clinically suspected or even accepted, but hitherto not experimentally demonstrated, aspects of the local humoral immune response in ocular toxoplasmosis. Thus, they contribute to our understanding of the disease and support our diagnostic attitude towards its laboratory confirmation.

Acknowledgement This study was supported by a grant from the Swiss National Science Foundation (no. 32-32757.91). 


\section{References}

1. Aouizerate F, Cazenave J, Poirier L, Verin P, Gervais C, Lagoutte F, Begueret J (1991) [Direct detection of toxoplasma in the aqueous humour by gene amplification]. J Fr Ophtalmol $14: 550-555$

2. Aouizerate F, Cazenave J, Poirier L, Verin P, Cheyrou A, Begueret J, Lagoutte F (1993) Detection of Toxoplasma gondii in aqueous humour by the polymerase chain reaction. $\mathrm{Br} \mathrm{J}$ Ophthalmol 77:107-109

3. Bloch-Michel E, Lambin P, Debbia M, Tounsi Y, Trichet C, Offret H (19971998) Local production of $\mathrm{IgG}$ and $\mathrm{IgG}$ subclasses in the aqueous humor of patients with Fuchs heterochromic cyclitis, herpetic uveitis and toxoplasmic chorioretinitis. Int Ophthalmol 21: 187-194

4. Bornand JE, de Gottrau P (1997)

Uveitis: is ocular toxoplasmosis only a clinical diagnosis? Ophthalmologica 211:87-89

5. Bosch-Driessen EH, Berendschot TT, Ongkosuwito JV, Rothova A (2002) Ocular toxoplasmosis: clinical features and prognosis of 154 patients. Ophthalmology 109:869-878

6. Couvreur J, Thulliez P (1996) Toxoplasmose acquise à localisation oculaire ou neurologique. Presse Med 25: 438-442

7. De Boer JH, Verhagen C, Bruinenberg M, Rothova A, Rothova A, de Jong PT, Baarsma GS, Van der Lelij A, Ooyman FM, Bollemeijer JG, Derhaag PJ, Kijlstra A (1996) Serologic and polymerase chain reaction analysis of intraocular fluids in the diagnosis of infectious uveitis. Am J Ophthalmol 121:650-658

8. De Jong PT (1989) Ocular toxoplasmosis; common and rare symptoms and signs. Int Ophthalmol 13:391-397

9. Derouin F, Lacroix C, Sumyuen MH, Romand S, Garin YJ (1995) Modèles expérimentaux de toxoplasmose. Applications pharmacologiques. Parasite 2:243-256

10. Desmonts G (1966) Definitive serological diagnosis of ocular toxoplasmosis. Arch Ophthalmol 76:839-851

11. Fardeau C, Romand S, Rao NA, Cassoux N, Bettembourg O, Thulliez P, Le Hoang P (2002) Diagnosis of toxoplasmic retinochoroiditis with atypical clinical features. Am J Ophthalmol 134:196-203
12. Fatoohi AF, Cozon GJ, Wallon M, Kah S, Gay-Andrieu F, Greenland T, Peyron F (2003) Cellular immunity to Toxoplasma gondii in congenitally infected newborns and immunocompetent infected hosts. Eur J Clin Microbiol Infect Dis 22:181-184

13. Fawzy M, Mahmoud LA, El Gindy AE, Hegab MA, Bahgat MA (1999) Value of estimating intraocular antibody production in diagnosis of typical and atypical lesions of ocular toxoplasmosis. J Egypt Soc Parasitol 29:735-743

14. Forrester JV, McMenamin PG (1999) Immunopathogenic mechanisms in intraocular inflammation. Chem Immuno 73:159-185

15. Gabrielian K, Oganesian A, Patel SC, Verp MS, Ernest JT (1999) Cellular response in rabbit eyes after human fetal RPE cell transplantation. Graefes Arch Clin Exp Ophthalmol 237: 326-335

16. Garweg J, Boehnke M, Koerner F (1996) Restricted applicability of the polymerase chain reaction for the diagnosis of ocular toxoplasmosis. Ger J Ophthalmol 5:104-108

17. Garweg JG (2005) Determinants of immunodiagnostic success in human ocular toxoplasmosis. Parasite Immunol 27:61-68

18. Garweg JG, Jacquier P, Flueckiger F (1998) Aktuelle Grenzen in der Diagnostik der okulären Toxoplasmose. Klin Monatsbl Augenheilkd 212: 330-333

19. Garweg JG, Kuenzli H, Boehnke M (1998) Experimental ocular toxoplasmosis in naive and primed rabbits. Ophthalmologica 212:136-141

20. Garweg JG, Jacquier P, Boehnke M (2000) Early aqueous humor analysis in patients with human ocular toxoplasmosis. J Clin Microbiol 38:996-1001

21. Garweg JG, Garweg SD, Flueckiger F, Jacquier P, Boehnke M (2004) Aqueous humor and serum immunoblotting for immunoglobulin GAME antibodies in human ocular toxoplasmosis. J Clin Microbiol 42:4593-4598

22. Goichot EL, Bloch-Michel E (1980) Intérêt de l'étude détaillée de la sérologie quantitative de l'humeur aqueuse pour le diagnostic de la toxoplasmose oculaire. A propos de 180 cas. J Fr Ophtalmol 3:21-25

23. Goldmann H, Witmer R (1954) Antikörper im Kammerwasser. Ophthalmologica 127:323-330
24. Hadjikouti CA, Wang Y, O'Rourke J, Cone RE (1995) Intracameral injection of antigen potentiates the production of antigen-specific $\mathrm{T}$ cell proteins in serum after the induction of delayed-type hypersensitivity. Invest Ophthalmol Vis Sci 36:1470-1476

25. Holliman RE, Stevens PJ, Duffy KT, Johnson JD (1991) Serological investigation in ocular toxoplasmosis. $\mathrm{Br} \mathbf{J}$ Ophthalmol 75:353-355

26. Jennis F (1966) Toxoplasmosis: an epidemiological study. Australas Ann Med 15:157-161

27. Johnson MW, Greven GM, Jaffe GJ, Sudhalkar H, Vine AK (1997) Atypical, severe toxoplasmic retinochoroiditis in elderly patients. Ophthalmology 104:48-57

28. Kijlstra A, Luyendijk L, Baarsma GS, Rothova A, Schweitzer CM, Timmerman Z, de Vries J, Breebaart AC (1989) Aqueous humor analysis as a diagnostic tool in Toxoplasma uveitis. Int Ophthalmol 13:383-386

29. Kijlstra A, Hoekzema R, van der Lelij A, Doekes G, Rothova A (1990) Humoral and cellular immune reactions against retinal antigens in clinical disease. Curr Eye Res 9 (Suppl):85-89

30. Kijlstra A, van den Horn GV, Luyendijk L, Baarsma GS, Schweitzer CM, Zaal MJ, Timmerman Z, Beintema M, Rothova A (1990) Laboratory tests in uveitis. New developments in the analysis of local antibody production. Doc Ophthalmol 75: 225-231

31. Klaren VN, Peek R (2001) Evidence for a compartmentalized B cell response as characterized by $\operatorname{IgG}$ epitope specificity in human ocular toxoplasmosis. J Immunol 167:6263-6269

32. Klaren VN, van Doornik CE, Ongkosuwito JV, Feron EJ, Kijlstra A (1998) Differences between intraocular and serum antibody responses in patients with ocular toxoplasmosis. Am J Ophthalmol 126:698-706

33. Labalette P, Delhaes L, Margaron F, Fortier B, Rouland JF (2002) Ocular toxoplasmosis after the fifth decade. Am J Ophthalmol 133:506-515

34. Langford MP, Orillac R, Chen D, Texada D (2003) Systemic and ocular antibody responses to inactivated acute hemorrhagic conjunctivitis (AHC) virus; enterovirus 70 (EV70). Ocul Immunol Inflamm 11:197-209 
35. Liekfeld A, Schweig F, Jaeckel C, Wernecke KD, Hartmann C, Pleyer U (2000) Intraocular antibody production in intraocular inflammation. Graefes Arch Clin Exp Ophthalmol 238: 222-227

36. McCannel CA, Holland GN, Helm CJ, Cornell PJ, Winston JV, Rimmer TG (1996) Causes of uveitis in the general practice of ophthalmology. UCLA Community-Based Uveitis Study Group. Am J Ophthalmol 121:35-46

37. Mets MB, Holfels E, Boyer KM, Swisher CN, Roizen N, Stein L, Stein M, Hopkins J, Withers S, Mack D, Luciano R, Patel D, Remington JS, Meier P, McLeod R (1996) Eye manifestations of congenital toxoplasmosis. Am J Ophthalmol 122:309-324

38. Moshfeghi DM, Dodds EM, Couto CA, Santos CI, Nicholson DH, Lowder CY, Davis JL (2004) Diagnostic approaches to severe, atypical toxoplasmosis mimicking acute retinal necrosis. Ophthalmology 111:716-725

39. Ongkosuwito JV, Feron EJ, van Doornik CE, Van der Lelij A, Hoyng CB, La Heij EC, Kijlstra A (1998) Analysis of immunoregulatory cytokines in ocular fluid samples from patients with uveitis. Invest Ophthalmol Vis Sci 39:2659-2665

40. Pedersen OO, Lorentzen-Styr AM (1981) Antibodies against Toxoplasma gondii in the aqueous humour of patients with active retinochoroiditis. Acta Ophthalmol (Copenh) 59: 719-726
41. Pelloux H, Mouillon M, Romanet JP, Reynier P, Ligeon P, Goullier-Fleuret A, Ambroise-Thomas P (1991) [Ocular toxoplasmosis. Comparison between two biological methods to study aqueous humour]. Presse Med 26: 1655-1658

42. Quentin CD, Reiber H (1997) Kammerwasseranalytik bei intraokularer Toxoplasmose. Ophthalmologe 94: 728-731

43. Quentin CD, Reiber H (2004) Fuchs heterochromic cyclitis: rubella virus antibodies and genome in aqueous humor. Am J Ophthalmol 138:46-54

44. Remky H (1968) Vergleichende Untersuchungen von Kammerwasser und Serum mittels des Dye-Tests. Klin Monatsbl Augenheilkd 153:617-623

45. Stanford MR, Gras L, Wade A, Gilbert RE (2002) Reliability of expert interpretation of retinal photographs for the diagnosis of toxoplasma retinochoroiditis. Br J Ophthalmol 86:636-639

46. Stanilova SA, Miteva LD, Tanchev SG (2005) Immunomodulatory effects of C3bgp on the antibody response to hemocyanin in outbred rabbits and the F1 generation of breeding with siblings. Vet Immunol Immunopathol 106:15-21

47. Streilein JW, Ohta K, Mo JS, Taylor AW (2002) Ocular immune privilege and the impact of intraocular inflammation. DNA Cell Biol 21:453-459

48. Tabbara KF (1995) Ocular toxoplasmosis: toxoplasmic retinochoroiditis. Int Ophthalmol Clin 35:15-29

49. Tanchev S, Gadjeva V, Stanilova S (2003) Influence of antigen stimulation on the oxidative stress parameters in outbred and inbred rabbits. Arch Physiol Biochem 111:437-442
50. Turunen HJ, Leinikki PO, Saari KM (1983) Demonstration of intraocular synthesis of immunoglobulin G toxoplasma antibodies for specific diagnosis of toxoplasmic chorioretinitis by enzyme immunoassay. J Clin Microbiol 17:988-992

51. Vallochi AL, Yamamoto JH, Schlesinger D, Machado MA, Silveira C, Martins MC, Belfort Jr R, Kalil J, Rizzo LV (2001) Lack of evidence for superantigen activity of Toxoplasma gondii towards human T cells. Braz J Med Biol Res 34:1023-1031

52. Villard O, Filisetti D, Roch-Deries F, Garweg J, Flament J, Candolfi E (2003) Comparison of enzyme-linked immunosorbent assay, immunoblotting, and PCR for diagnosis of toxoplasmic chorioretinitis. J Clin Microbiol 41:3537-3541

53. Vinhal FA, Pena JD, Katina JH, Brandao EO, Silva DA, Orefice F, Mineo JR (1994) Analysis of aqueous humor in ocular toxoplasmosis: detection of low avidity IgG specific to Toxoplasma gondii. Appl Parasitol 35:1-7

54. Yamamoto JH, Vallochi AL, Silveira C, Filho JK, Nussenblatt RB, Cunha-Neto E, Gazzinelli RT, Belfort Jr R, Rizzo LV (2000) Discrimination between patients with acquired toxoplasmosis and congenital toxoplasmosis on the basis of the immune response to parasite antigens. J Infect Dis 181:2018-2022 